\title{
Modelos psicosociales de responsabilidad social en universitarios de diferentes campus
}

Mario-Gerardo Serrano-Pereira, María-Teresita Castillo-León y Emelia Hernández-Payán

\section{RESUMEN}

En este artículo se desarrollan modelos para explicar la responsabilidad social en estudiantes de diferentes áreas del conocimiento de una universidad pública del sureste mexicano. Participaron 500 estudiantes elegidos al azar. Se les administraron instrumentos para medir locus de control, autoconcepto, enfrentamiento a los problemas, empatía, salud mental positiva y responsabilidad social. Los resultados mostraron diferencias significativas en todas las variables por área del conocimiento. Asimismo, los modelos psicosociales resultantes evidenciaron la contribución del autoconcepto, el enfrentamiento y la empatía para la responsabilidad social universitaria. Los resultados se analizan a partir de la congruencia con el enfoque de la responsabilidad social universitaria.

Palabras clave: responsabilidad social, formación universitaria, factores psicosociales, modelos, educación superior, México.

Mario-Gerardo Serrano-Pereira 


\section{Modelos psicossociais de responsabilidade social em universitários de diferentes campus}

\section{RESUMO}

Neste artigo se desenvolvem modelos para explicar a responsabilidade social em estudantes de diferentes áreas do conhecimento de uma universidade pública do sudeste mexicano. Participaram 500 estudantes elegidos aleatoriamente. Administraram-se instrumentos para medir locus de controle, autoconceito, enfrentamento aos problemas, empatia, saúde mental positiva e responsabilidade social. Os resultados mostraram diferenças significativas em todas as variáveis por área de conhecimento. Além disso, os modelos psicossociais resultantes evidenciaram a contribuição do autoconceito, o enfrentamento e a empatia para a responsabilidade social universitária. Os resultados se analisam a partir da congruência com o enfoque da responsabilidade social universitária.

Palavras chave: responsabilidade social, formação universitária, fatores psicossociais, modelos, educação superior, México.

\section{Psychosocial models of social responsibility in university students from different campuses}

\section{ABSTRACT}

This article develops models to explain social responsibility in students from different areas of knowledge at a public university in southeastern Mexico. Five hundred randomly selected students took part in this project. Instruments were administered to measure locus of control, self-concept, coping with problems, empathy, positive mental health and social responsibility. The results showed significant differences in all variables by knowledge area. Likewise, the resulting psychosocial models evidenced the contribution of self-concept, coping and empathy to university social responsibility. The results are analyzed on the basis of congruence with the university social responsibility approach.

Key words: social responsibility, university education, psychosocial factors, psychosocial models, higher education, Mexico. 


\section{Introducción}

Actualmente la contribución de las instituciones de educación superior (IES) es fundamental para el alcance de los objetivos de desarrollo social, económico, tecnológico y sostenible (ANUIES, 2018; Domínguez y Rama, 2012; Larrán-Jorge y Andrades-Peña, 2015; Martí et al., 2018; Ruiz-Corbella y López-Gómez, 2019). Las expectativas son tan altas como los desafíos. Gobiernos, empresas y sociedad civil demandan de las universidades nuevos profesionales adecuadamente formados tanto en el ámbito profesional como en lo personal. Es decir, se considera que el universitario de hoy debe contar con las herramientas teórico-prácticas de la disciplina que haya estudiado, además de una serie de características psicosociales que le den perspectiva social y responsable a su persona. La interacción de los elementos profesionales y humanos, como resultado de la educación universitaria, articula los antiguos ideales, todavía vigentes, en torno a los efectos de la educación en los seres humanos (saber conocer, saber hacer, saber ser) (ANUIES, 2018; UNESCO, 2013).

Como respuesta a las expectativas que la sociedad tiene respecto al rol para el desarrollo que desempeña la universidad, surge el enfoque de la responsabilidad social, el cual le da pertinencia y transcendencia social a las funciones universitarias relativas a la formación profesional y ciudadana de estudiantes; a la generación, aplicación y difusión del conocimiento a partir de las problemáticas prioritarias, así como a la necesaria y responsable vinculación de la universidad con la sociedad (Martínez-Usarralde et al., 2019; Nieves, 2010; Olarte-Mejía y Ríos-Osorio, 2015; Vallaeys, 2014).

Por tanto, la responsabilidad social (RS) es una dimensión importante que configura y da sentido a la persona formada en una IES. Se parte de la idea que sostiene que el estudiante universitario, durante su trayectoria académica, toma conciencia de la realidad que le rodea, se posiciona frente a ella, identifica y prioriza los desafios que la realidad (social, económica, medio ambiental, etcétera) impone y que, con las herramientas teórico-metodológicas y psicosociales que ha adquirido y/o fortalecido como resultado de su educación, estudia, interviene y promueve el cambio frente a las problemáticas identificadas de manera uni, multi e interdisciplinaria (Castillo León, 2016; Rivera, 2018; Rodríguez, 2012; Ruiz-Corbella y López-Gómez, 2019). Por lo anterior, los estudiantes son el resultado de un complejo y trascendente proceso formativo que, al final, los orienta para ser personas integrales, activas y participativas frente al momento histórico que toca vivir.

\section{Conceptualización de responsabilidad social universitaria y su relación con variables psicosociales diversas}

Es claro que, dadas las condiciones del mundo actual en materia de desigualdad, pobreza, violencia, salud, alimentación, empleo, problemas medio-ambientales (ONU, 2015; UNESCO, 2013), la formación de profesionales socialmente responsables con conciencia ciudadana es fundamental. Logarlo no es sólo cuestión de transmitir información, sino de un proceso formativo integral donde la adquisición y fortalecimiento de diversas características psicosociales resultan importantes.

De acuerdo con Vallaeys (2014) una persona socialmente responsable es aquella que ha tomado conciencia de la realidad que el mundo enfrenta en materia de derechos humanos, construcción de una sociedad civil organizada, lucha contra la corrupción, desarrollo económico con rostro humano, exigencia de la calidad en las organizaciones públicas y privadas, lucha contra la pobreza, protección del medio ambiente y el desarrollo global sostenible y equitativo. A partir de lo anterior, se deduce que conceptualmente el enfoque de la responsabilidad social universitaria (RSU) implica una serie de características psicosociales que denotan una forma de ser y de actuar de la persona considerada como socialmente responsable. Si bien la investigación 
e implementación del enfoque está en sus inicios (ANUIES, 2018), algunos estudios han aportado evidencia en torno a la vinculación existente, tanto a nivel teórico como empírico, entre la responsabilidad social y algunas variables psicosociales como la empatía (Arango et al., 2014; Martí, 2011; Martí et al., 2014), el autoconcepto (Esnaola et al., 2008; Goñí, 2009), el locus de control (Mayorá-Pernía y Fernández, 2015; Pinto, 2000; Serrano, 2003; Velasco et al., 2015), el enfrentamiento a los problemas (Góngora, 2000; Góngora et al., 2008) y la salud mental positiva (Barrera y Flores, 2015; Castillo León, 2016; Lluch, 1999). Cada uno de los estudios anteriores ha explorado, por separado, la asociación entre la responsabilidad social con cada una de las variables antes mencionadas. Sin embargo, se considera también necesario realizar estudios que vinculen a varias de estas características psicosociales en un modelo que pueda explicar la responsabilidad social en los universitarios.

A partir de lo anterior, el presente trabajo se planteó como propósito precisar aquellas variables psicosociales que, en su conjunto, explican la responsabilidad social en estudiantes universitarios del sureste de México, particularmente de la Universidad Autónoma de Yucatán (UAY). Asimismo, a partir de las variables identificadas, elaborar modelos psicosociales explicativos de la responsabilidad social para estudiantes de diferentes campus de dicha universidad. Para este trabajo se eligieron algunas de las variables psicosociales mayormente estudiadas en psicología y educación como el autoconcepto, la empatía, el enfrentamiento a los problemas, el locus de control y la salud mental desde la perspectiva positiva.

\section{Método}

Siendo una investigación de tipo cuantitativa y no experimental, se procedió mediante un diseño de investigación transeccional explicativo, correlacionalcausal (Hernández, Fernández y Baptista, 2014), que tuvo como objetivo construir modelos psicosociales para explicar la responsabilidad social en universitarios de diferentes campus; adicionalmente, se buscó conocer si existen diferencias entre los estudiantes por campus en cada una de las variables estudiadas.

Los datos fueron capturados y procesados a través del IBM SPSS Statistics versión 19, en el cual se realizaron cuatro tipos de análisis: 1) análisis descriptivos a través de frecuencias, porcentajes, medidas de tendencia central y de dispersión en las variables; 2) análisis psicométricos de las diferentes escalas utilizadas, tales como la validación factorial por medio del análisis factorial y el análisis de la consistencia interna por medio del coeficiente alpha de Cronbach, lo cual permitió verificar la confiabilidad de los instrumentos; 3) análisis inferenciales para probar diferencias entre las variables a través del análisis de varianza (ANOVA), y 4) análisis de regresión múltiple con el fin de establecer las variables predictoras de la responsabilidad social, lo que permitió construir los modelos explicativos de la responsabilidad social a partir de las variables psicosociales estudiadas.

\section{Sujetos}

Participaron 500 estudiantes de la Universidad $\mathrm{Au}-$ tónoma de Yucatán (UAY), México pertenecientes a los cinco campus que conforman dicha universidad (ciencias sociales, ciencias exactas, ciencias de la salud, ciencias bioagropecuarias y arquitectura-hábitat-arte-diseño). El 50\% de la muestra conformada por mujeres y el 50\% restante por varones. Fueron seleccionados al azar a partir de las facultades en las que se encontraban inscritos. El rango de edad varió de los 18 a los 31 años, siendo los 19 años la edad más frecuente (25.6\%). En cuanto al grado escolar, fluctuó del primero al quinto grado de la carrera, siendo la mayoría del primero $(41.8 \%)$ y del segundo año $(21.6 \%)$. En la siguiente figura se presenten las principales características sociodemográficas de la muestra. 
Figura 1. Características sociodemográficas de la muestra

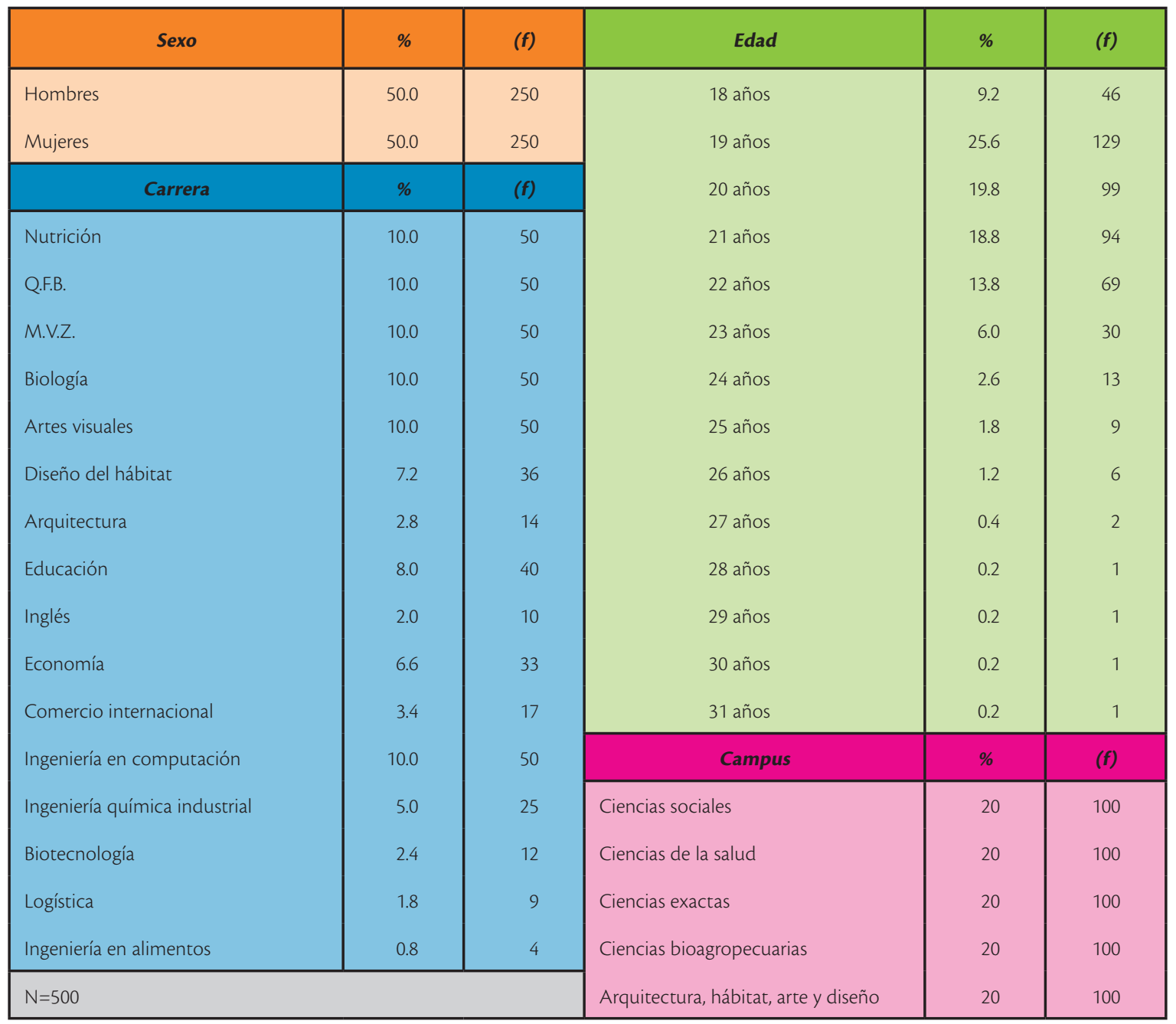

Fuente: elaboración propia.

\section{Instrumentos}

\section{Locus de control}

Se utilizó la versión corta de 12 reactivos Likert de la escala multidimensional de locus de control (La Rosa, 1986) que mide la idea de la persona sobre los factores (internos o externos) que controlan su vida a través de cuatro opciones de respuesta que van de uno (totalmente en desacuerdo) a cuatro (totalmente de acuerdo). El análisis factorial en la muestra de universitarios reveló la existencia de tres dimensiones que explicaron el $56.35 \%$ de la varianza. Tales dimensiones fueron nombradas y definidas de acuerdo con La Rosa (1986).
La primera dimensión es la Suerte $(\alpha=0.77)$, que se refiere a considerar que la suerte, por sobre la inteligencia, determina los éxitos; la segunda, Internalidad $(\alpha=$ 0.69) que alude a pensar que los factores internos como el esfuerzo determinan el éxito y, finalmente la tercera, Afectividad $(\alpha=0.70)$ que se refiere a considerar que la simpatía y las relaciones afectivas constituyen los factores que mayormente influyen en los éxitos.

\section{Autoconcepto}

Se midió a través de la escala mexicana de autoconcepto (Díaz-Loving et al., 2002) la cual mide la 
percepción del sujeto hacia su propio yo a través de 44 reactivos tipo Likert pictórico con siete opciones de respuesta que van de uno (nada de la característica) a siete (mucho de la característica). El análisis factorial reveló la existencia de nueve dimensiones, sin embargo, se seleccionaron únicamente las primeras ocho por ofrecer una mayor claridad conceptual. Las dimensiones seleccionadas explicaron el $57.47 \%$ de la varianza y fueron definidas de acuerdo con DíazLoving et al. (2002): Social expresivo $(\alpha=0.86)$, se refiere a las características positivas del individuo que le permiten comunicarse y expresarse en su medio social; Social afiliativo $(\alpha=0.90)$ hace referencia a los sentimientos positivos interindividuales experimentados por el sujeto, donde la otra persona es el objeto de acercamiento y afecto; Depresivo $(\alpha=$ 0.80) son las características del sujeto que demuestran nostalgia y alteración, indicando un posible deterioro en la salud emocional; Inteligencia socioemocional $(\alpha=0.80)$, define a la persona en forma socioemocional ecuánime y flexible para enfrentar las relaciones interpersonales y los problemas de la vida; Emotivo-negativo-autoafirmativo ( $\alpha=0.71)$, incluye las características negativas temperamentales que implican inconformidad, impulsividad y exaltación de las emociones; Instrumental constructivo $(a=0.64)$, son las características que reflejan funcionalidad y habilidades en diferentes ámbitos como el trabajo, la escuela o el hogar; Ético normativo ( $a=0.72$ ), serie de características que demuestran congruencia con los valores socio-personales que reflejan los mandatos de la cultura y, finalmente, Control externo-negativopasivo ( $\alpha=0.75$ ), que son las características negativas que demuestran incapacidad y desinterés para actuar constructivamente ante el medio.

\section{Enfrentamiento a los problemas}

Se utilizó la subescala vida de la escala multidimensional y multisituacional de enfrentamiento a los problemas (Góngora y Reyes-Lagunes, 1998), que mide el enfrentamiento ante los problemas como un rasgo estable y duradero en la vida. Consta de 18 reactivos tipo Likert pictórico con siete opciones de respuesta que van de siempre (siete) a nunca (uno). El análisis factorial encontró cinco dimensiones, sin embargo, sólo se seleccionaron las primeras cuatro debido a que ofrecieron mayor claridad conceptual. Las dimensiones seleccionadas explicaron el $54.56 \%$ de la varianza y se definieron según Góngora y Reyes-Lagunes (1998): Directo revalorativo ( $a=0.83$ ), significa que ante los problemas de la vida, la persona intenta hacer algo para resolverlos, tratando de aprender o ver lo positivo; Emocional negativo $(\alpha=0.74)$, cuando la persona expresa sólo tener una emoción que no lleva directamente a la solución del problema; Apoyo social $(\alpha=0.77)$, implica que la persona comparta con alguien el problema o la búsqueda de su solución, y Evasivo $(\alpha=0.59)$, significa que la persona evite, escape o minimice el problema y su solución.

\section{Empatía}

Se midió a través del Índice de reactividad interpersonal (Davis, 1980 en Mart́, 2011), el cual consta de 28 reactivos distribuidos en cuatro subescalas de siete reactivos Likert cada una, que miden dimensiones cognitivas y emocionales de la empatía. Cuenta con cinco opciones de respuesta que van de cero (no me describe bien) a cuatro (me describe muy bien). El análisis factorial reveló la existencia de cuatro dimensiones, que explicaron el $39.47 \%$ de la varianza y se definieron de acuerdo con Martí (2011): Malestar personal $(\alpha=0.77)$, se refiere a la experiencia de malestar que siente una persona ante el estado emocional de otra, dirigiéndola a intentar aliviar su propio estado en lugar de ayudar a la otra persona; Fantasía $(\alpha=0.79$ ), capacidad imaginativa para identificarse y ponerse en el lugar de personajes de ficción; Preocupación empática $(\alpha=0.59)$, respuesta afectiva ante las dificultades de otras, manifestándose en sentimientos de compasión y cariño hacia otros; Toma de perspectiva $(a=0.66)$, adopción del punto de vista de otra persona ante situaciones presentes o hipotéticas. 


\section{Salud mental positiva}

Se utilizó la escala de Lluch (1999) que consta de 39 reactivos Likert con cuatro opciones de respuesta que van de uno (siempre o casi siempre) a cuatro (nunca o casi nunca). El análisis factorial encontró ocho dimensiones de las cuales se seleccionaron las primeras siete por ofrecer una mayor claridad conceptual. Las dimensiones seleccionadas explicaron el 51.29\% de la varianza y fueron definidas de acuerdo con Lluch (1999) como: Satisfacción personal $(\alpha=0.84)$, que hace referencia a que la persona cuente con autoconcepto, satisfacción con la vida personal y satisfacción con las perspectivas futuras; Resolución de problemas $(\alpha=0.78)$, capacidad para analizar y tomar decisiones; Autocontrol $(a=0.80)$, capacidad para el afrontamiento de situaciones conflictivas con equilibrio, control emocional, tolerancia a la ansiedad y al estrés; Autoactualización ( $\alpha=0.78$ ), actitud de crecimiento y desarrollo personal contínuo; Autonomía $(\alpha=0.66)$, capacidad para tener criterio propio, independencia, autorregulación de la propia conducta, seguridad personal y confianza en sí mismo; Actitud prosocial $(a=0.64)$, actitud de ayuda, empatía, apoyo y altruismo hacia los demás, y Habilidades de relación interpersonal ( $a=0.68$ ), capacidad para establecer relaciones interpersonales, generar empatía, entender los sentimientos de los demás, para dar apoyo y para establecer relaciones interpersonales íntimas.

\section{Responsabilidad social}

Escala elaborada para el presente estudio, consta de 30 reactivos tipo Likert con cuatro opciones de respuestas que van de uno (totalmente en desacuerdo) a cuatro (totalmente de acuerdo). Mide la percepción del estudiante para responder en forma socialmente responsable en las cuatro dimensiones del modelo de RS de la UAY. El análisis factorial reveló la existencia de cuatro dimensiones que explicaron el $41.09 \%$ de la varianza, llamadas: Participación social $(\alpha=$ 0.75), que se refiere a la capacidad y disposición del universitario para participar y desarrollar proyectos promotores del desarrollo comunitario, la calidad de vida, el cuidado del ambiente así como para participar en la formulación de políticas públicas; Gestión social del conocimiento $(\alpha=0.76)$, que se refiere a la capacidad del universitario para generar, difundir y aplicar el conocimiento en beneficio de su comunidad contribuyendo a la solución de problemáticas de su entorno; Gestión responsable ( $\alpha=0.72$ ), que alude a la percepción que el estudiante tiene de su universidad como una institución caracterizada por un adecuado clima organizacional que promueve entre sus estudiantes la conducta ética y el respeto por los derechos humanos; Formación profesional y ciudadana $(\alpha=0.62)$, que se refiere a la aportación que el estudiante nota de sus estudios universitarios y que lo transforman en una persona ética capaz de cuidar de sí mismo, de los demás, del medio ambiente y que le posibilita contribuir al desarrollo sustentable global.

\section{Procedimiento}

La aplicación se llevó a cabo de manera colectiva en los salones de clase, o bien en las áreas asignadas por las autoridades de las distintas facultades. Durante la aplicación se respetaron los principios éticos de consentimiento informado, derecho a la información, protección de datos personales, garantías de confidencialidad, no discriminación y gratitud. La duración de la aplicación fue de 30 minutos en promedio.

\section{Resultados}

Los análisis descriptivos realizados en los factores de las escalas revelaron lo siguiente. En cuanto al locus de control, los universitarios expresaron un locus interno $(\mathrm{M}=3.65)$ más que externo, basado en la suerte $(\mathrm{M}=1.65)$ o en su simpatía $(\mathrm{M}=2.38)$. Con respecto al autoconcepto los universitarios expresaron una imagen positiva de sí en términos sociales expresivos $(M=5.27)$, sociales afiliativos $(M=5.17)$, inteligentes en el ámbito socioemocional $(\mathbf{M}=5.24)$, instrumentales constructivos $(M=5.13)$ y bastante éticos $(\mathrm{M}=6.18)$. Por otro lado, consideran que los aspectos 
negativos no forman parte de su autoconcepto, por ejemplo, consideran poseer poco control externo negativo pasivo (M=2.72), ni poco ni mucho autoconcepto emotivo negativo $(\mathrm{M}=3.88)$ y ni poco ni mucho autoconcepto depresivo $(\mathrm{M}=4.18)$. En cuanto a los estilos que tienen para enfrentar los problemas de la vida mencionan que, con bastante frecuencia, enfrentan directamente los problemas y aprenden algo en el proceso $(\mathrm{M}=5.76)$, la mayoría de las veces buscan apoyo social $(\mathrm{M}=4.35)$ o solamente expresan emociones negativas $(\mathrm{M}=4.54)$ y que, algunas veces, sólo evaden los problemas que se les presentan ( $M=3.85)$. Con respecto a la empatía, los universitarios consideran que les describe bien tanto la preocupación empática $(\mathrm{M}=3.60)$ como la toma de perspectiva $(M=3.25)$. Por otro lado, consideran que poco les describe tanto el malestar emocional $(\mathrm{M}=2.42)$ como la fantasía $(M=2.85)$. En cuanto a la salud mental positiva, mencionaron que algunas veces están satisfechos con ellos mismos $(M=3.15)$, son resolutivos ante los problemas $(\mathrm{M}=3.05)$, tienden al autocontrol $(\mathbf{M}=2.71)$, a la autoactualización ( $\mathbf{M}=3.20)$, a la autonomía (M=2.91) y a la actitud prosocial ( $\mathrm{M}=2.99)$ y que algunas veces son hábiles en las relaciones interpersonales $(M=2.87)$. Con respecto a la responsabilidad social universitaria, mencionan que están de acuerdo con la formación profesional y ciudadana que están recibiendo de su universidad ( $\mathrm{M}=3.12)$ pero en desacuerdo con la capacidad para la gestión social de su conocimiento $(\mathrm{M}=2.42)$, para la participación social $(\mathrm{M}=2.75)$ y respecto a la gestión responsable de su universidad ( $\mathrm{M}=2.88$ ) (tabla 1).

Los análisis de diferencias por tipo de campus revelaron resultados estadísticamente significativos, aunque en todos los casos, las diferencias en las medias resultaron mínimas y los puntajes obtenidos, cercanos a la media teórica. Se encontraron diferencias estadísticamente significativas en las tres dimensiones del locus de control. Tanto en el factor suerte $\left(\mathrm{F}_{(4,495)}=2.78, \mathrm{p}=0.02\right)$ como en el el factor afectividad $\left(\mathrm{F}_{(4,495)}=2.98, \mathrm{p}=0.01\right) \operatorname{los}$ puntajes más altos los obtuvieron los estudiantes de ciencias exactas $(\mathrm{M}=1.73$ y $\mathrm{M}=2.54)$; mientras que en el factor internalidad $\left(\mathrm{F}_{(4,495)}=4.19, \mathrm{p}=0.001\right) \mathrm{el}$ puntaje más alto lo obtuvo el grupo de estudiantes de ciencias biológicas y agropecuarias $(\mathrm{M}=3.77)$.

En autoconcepto, las diferencias se observaron en los factores social expresivo $\left(\mathrm{F}_{(4,495)}=3.53, \mathrm{p}=.001\right)$, social afiliativo $\left(\mathrm{F}_{(4,495)}=4.13, \mathrm{p}=0.001\right) \mathrm{y}$ en emotivo-negativo-autoafirmativo $\left(\mathrm{F}_{(4,495)}=3.02, \mathrm{p}=0.01\right)$, en los tres casos las medias más elevadas las obtuvieron los estudiantes de ciencias sociales ( $\mathrm{M}=$ $5.48, \mathrm{M}=5.59$ y $\mathrm{M}=4.09)$. Por su parte, los estudiantes del campus de ciencias biológicas y agropecuarias obtuvieron los puntajes más elevados en inteligencia sociocemocional $\left(\mathrm{F}_{(4,495)}=2.91, \mathrm{p}=0.02 ; \mathrm{M}=5.40\right)$ $\mathrm{y}$ en la dimensión ética normativa $\left(\mathrm{F}_{(4,495)}=3.20\right.$, $\mathrm{p}=0.01 ; \mathrm{M}=6.29)$. Finalmente, en la dimensión del autoconcepto referida al control externo-pasivo-negativo, los estudiantes de ciencias exactas obtuvieron la media más alta $\left(\mathrm{F}_{(4,495)}=2.62, \mathrm{p}=0.03 ; \mathrm{M}=2.92\right)$.

Se encontró también una diferencia estadísticamente significativa en el estilo de enfrentamiento emocional negativo $\left(\mathrm{F}_{(4,495)}=4.44, \mathrm{p}=0.001\right)$, en donde los estudiantes del campus de ciencias sociales obtuvieron la media más alta $(\mathrm{M}=4.80)$.

En cuanto a la empatía, se encontraron diferencias estadísticamente significativas en malestar personal $\left(\mathrm{F}_{(4,495)}=3.51, \mathrm{p}=0.008\right)$ y en preocupación empática $\left(\mathbf{F}_{(4,495)}=3.58, \mathrm{p}=0.007\right)$. En malestar emocional los estudiantes de ciencias sociales obtuvieron la media más alta $(M=2.62)$ mientras que en preocupación empática los estudiantes de ciencias bioagropecuarias obtuvieron el puntaje más alto $(\mathrm{M}=3.70)$. El único factor de la salud mental positiva que marcó una diferencia por campus fue el autocontrol $\left(\mathrm{F}_{(4,495)}=\right.$ $2.69, \mathrm{p}=0.03)$ en donde los estudiantes de ciencias exactas obtuvieron la media más alta $(\mathrm{M}=2.83)$.

Finalmente, los cuatro factores de la responsabilidad social universitaria mostraron diferencias en los estudiantes de los diferentes campus. En participación social $\left(\mathrm{F}_{(4,495)}=2.85, \mathrm{p}=0.02\right)$, gestión social del conocimiento $\left(\mathrm{F}_{(4,495)}=4.18, \mathrm{p}=0.002\right)$ y en gestión 
Tabla 1. Consistencia interna, medias y desviaciones estándar para los factores de las escalas

\begin{tabular}{|c|c|c|c|c|}
\hline Escalas & Factores & Media & Desviación estándar & alpha \\
\hline \multirow{3}{*}{ Locus de control } & Suerte & 1.65 & 0.52 & 0.77 \\
\hline & Internalidad & 3.65 & 0.43 & 0.69 \\
\hline & Afectividad & 2.38 & 0.56 & 0.70 \\
\hline \multirow{8}{*}{ Autoconcepto } & Social expresivo & 5.27 & 1.00 & 0.86 \\
\hline & Social afiliativo & 5.17 & 1.36 & 0.90 \\
\hline & Depresivo & 4.18 & 1.19 & 0.80 \\
\hline & Inteligencia socioemocional & 5.24 & 1.06 & 0.80 \\
\hline & Emotivo-negativo-autoafirmativo & 3.88 & 1.11 & 0.71 \\
\hline & Instrumental constructivo & 5.13 & 1.04 & 0.64 \\
\hline & Ético normativo & 6.18 & 0.74 & 0.72 \\
\hline & Control-externo-negativo-pasivo & 2.72 & 1.04 & 0.75 \\
\hline \multirow{4}{*}{$\begin{array}{l}\text { Enfrentamiento a los } \\
\text { problemas }\end{array}$} & Directo revalorativo & 5.76 & 0.91 & 0.83 \\
\hline & Emocional negativo & 4.54 & 1.20 & 0.74 \\
\hline & Apoyo social & 4.35 & 1.42 & 0.77 \\
\hline & Evasivo & 3.85 & 1.23 & 0.59 \\
\hline \multirow{4}{*}{ Empatía } & Malestar personal & 2.42 & 0.76 & 0.77 \\
\hline & Fantasía & 2.85 & 1.03 & 0.79 \\
\hline & Preocupación empática & 3.60 & 0.70 & 0.59 \\
\hline & Toma de perspectiva & 3.25 & 0.89 & 0.66 \\
\hline \multirow{7}{*}{ Salud mental positiva } & Satisfacción personal & 3.15 & 0.69 & 0.84 \\
\hline & Resolución de problemas & 3.05 & 0.66 & 0.78 \\
\hline & Autocontrol & 2.71 & 0.72 & 0.80 \\
\hline & Autoactualización & 3.20 & 0.60 & 0.78 \\
\hline & Autonomía & 2.91 & 0.80 & 0.66 \\
\hline & Actitud prosocial & 2.99 & 0.55 & 0.64 \\
\hline & Habilidades de relación interpersonal & 2.87 & 0.69 & 0.68 \\
\hline \multirow{4}{*}{ Responsabilidad social } & Participación social & 2.75 & 0.58 & 0.75 \\
\hline & Gestión social del conocimiento & 2.42 & 0.65 & 0.76 \\
\hline & Gestión responsable & 2.88 & 0.68 & 0.72 \\
\hline & Formación profesional y ciudadana & 3.12 & 0.53 & 0.62 \\
\hline
\end{tabular}

Fuente: elaboración propia. 
responsable $\left(\mathrm{F}_{(4,495)}=6.39, \mathrm{p}=0.001\right)$ los estudiantes de ciencias sociales obtuvieron los puntajes más altos $(\mathrm{M}=2.88, \mathrm{M}=2.61$ y $\mathrm{M}=3.05)$. Pero, en formación profesional y ciudadana $\left(\mathrm{F}_{(4,495)}=12.15, \mathrm{p}=0.001\right)$ los estudiantes de ciencias bioagropecuarias obtuvieron la media más alta $(\mathrm{M}=3.77)$ (tabla 2$)$.

Tabla 2. Análisis de varianza en los factores de las diferentes escalas por campus

\begin{tabular}{|c|c|c|c|c|c|c|c|c|}
\hline \multirow{2}{*}{ 空 } & \multirow[b]{2}{*}{ Factores } & \multicolumn{5}{|c|}{ Campus } & \multirow[b]{2}{*}{$f$} & \multirow[b]{2}{*}{$p$} \\
\hline & & Salud & $\begin{array}{l}\text { Bioagro- } \\
\text { pecuarias }\end{array}$ & Sociales & Exactas & $\begin{array}{l}\text { Arq/hábitat/ } \\
\text { arte/diseño }\end{array}$ & & \\
\hline \multirow{3}{*}{ 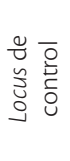 } & Suerte & 1.67 & 1.52 & 1.62 & 1.73 & 1.72 & 2.78 & $0.02^{*}$ \\
\hline & Internalidad & 3.68 & 3.77 & 3.67 & 3.56 & 3.57 & 4.19 & $0.001^{* * *}$ \\
\hline & Afectividad & 2.32 & 2.31 & 2.33 & 2.54 & 2.38 & 2.98 & $0.01^{* *}$ \\
\hline \multirow{8}{*}{ 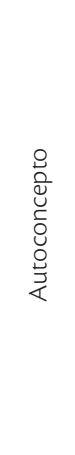 } & Social expresivo & 5.31 & 5.25 & 5.48 & 5.35 & 4.97 & 3.53 & $0.001^{* * *}$ \\
\hline & Social afiliativo & 5.21 & 5.12 & 5.59 & 5.08 & 4.84 & 4.13 & $0.001^{* * *}$ \\
\hline & Depresivo & 4.18 & 3.98 & 4.20 & 4.09 & 4.43 & 1.98 & 0.09 \\
\hline & Inteligencia socioemocional & 5.32 & 5.40 & 5.21 & 5.35 & 4.95 & 2.91 & $0.02^{*}$ \\
\hline & Emotivo-negativo autoafirmativo & 3.97 & 3.68 & 4.09 & 3.67 & 3.99 & 3.02 & $0.01^{* *}$ \\
\hline & Instrumental constructivo & 5.25 & 5.07 & 5.20 & 5.16 & 4.98 & 1.02 & 0.39 \\
\hline & Ético normativo & 6.26 & 6.29 & 6.27 & 6.08 & 6.00 & 3.20 & $0.01^{* *}$ \\
\hline & Control externo- negativo-pasivo & 2.60 & 2.57 & 2.61 & 2.92 & 2.88 & 2.62 & $0.03^{*}$ \\
\hline \multirow{4}{*}{ 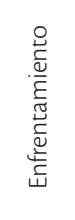 } & Directo revalorativo & 5.87 & 5.79 & 5.76 & 5.87 & 5.53 & 2.25 & 0.06 \\
\hline & Emocional negativo & 4.57 & 4.36 & 4.80 & 4.22 & 4.76 & 4.44 & $0.001^{* * *}$ \\
\hline & Apoyo social & 4.20 & 4.39 & 4.55 & 4.33 & 4.27 & 0.86 & 0.48 \\
\hline & Evasivo & 3.73 & 3.76 & 3.94 & 4.00 & 3.83 & 0.93 & 0.44 \\
\hline \multirow{4}{*}{$\frac{\sqrt[T]{\tilde{N}}}{\stackrel{\sim}{E}}$} & Malestar personal & 2.32 & 2.35 & 2.62 & 2.30 & 2.51 & 3.51 & $0.008^{* *}$ \\
\hline & Fantasía & 2.72 & 2.79 & 3.03 & 2.74 & 2.98 & 1.91 & 0.107 \\
\hline & Preocupación empática & 3.57 & 3.70 & 3.68 & 3.39 & 3.67 & 3.58 & $0.007^{* *}$ \\
\hline & Toma de perspectiva & 3.15 & 3.36 & 3.17 & 3.40 & 3.18 & 1.72 & 0.144 \\
\hline \multirow{7}{*}{ 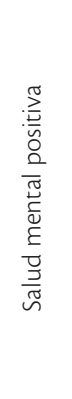 } & Satisfacción personal & 3.22 & 3.22 & 3.16 & 3.02 & 3.12 & 1.52 & 0.19 \\
\hline & Resolución de problemas & 3.16 & 3.08 & 3.02 & 3.04 & 2.95 & 1.63 & 0.16 \\
\hline & Autocontrol & 2.77 & 2.74 & 2.69 & 2.83 & 2.52 & 2.69 & $0.03^{*}$ \\
\hline & Autoactualización & 3.22 & 3.28 & 3.20 & 3.15 & 3.16 & 0.79 & 0.52 \\
\hline & Autonomía & 2.99 & 3.04 & 2.80 & 2.87 & 2.87 & 1.51 & 0.19 \\
\hline & Actitud prosocial & 3.04 & 3.07 & 2.97 & 2.94 & 2.96 & 1.05 & 0.38 \\
\hline & Habilidades de relación interpersonal & 2.87 & 2.81 & 3.00 & 2.87 & 2.80 & 1.25 & 0.28 \\
\hline \multirow{4}{*}{ 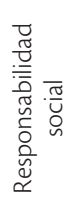 } & Participación social & 2.77 & 2.78 & 2.88 & 2.61 & 2.72 & 2.85 & $0.02^{*}$ \\
\hline & Gestión social del conocimiento & 2.41 & 2.44 & 2.61 & 2.23 & 2.44 & 4.18 & $0.002^{* *}$ \\
\hline & Gestión responsable & 2.95 & 3.01 & 3.05 & 2.70 & 2.71 & 6.39 & $0.001^{* * *}$ \\
\hline & Formación profesional y ciudadana & 3.22 & 3.77 & 3.12 & 2.95 & 2.95 & 12.15 & $0.001^{* * *}$ \\
\hline
\end{tabular}

Fuente: elaboración propia. ${ }^{*} p \leq 0.05,{ }^{* *} p \leq 0.01,{ }^{* * *} p \leq 0.001$. 
Para desarrollar los modelos psicosociales de la responsabilidad social se realizaron análisis de regresión múltiple para cada grupo de estudiantes de los diferentes campus. Se consideraron como variables independientes las 26 dimensiones obtenidas en los análisis factoriales de los instrumentos utilizados. Cabe mencionar que, para dichos análisis, se consideró a la responsabilidad social universitaria como una variable global que incluyó a las cuatro dimensiones previamente detectadas (participación social, gestión social del conocimiento, gestión responsable, y formación profesional y ciudadana).

A continuación, se presentan los resultados de los análisis de regresión múltiple para los estudiantes de los diferentes campus. En cada análisis de regresión se reporta la ecuación final (regresión), así como también la correlación múltiple $(\mathrm{R})$, el coeficiente de determinación $\left(\mathrm{R}^{2}\right)$, el coeficiente de determinación ajustado $\left(\mathrm{R}^{2}\right.$ aj.) y el error estándar de estimación obtenido (EE); se señala el análisis de varianza $(\mathrm{F})$ y su significancia respectiva. Se reportan las dimensiones que entraron al modelo de regresión en la ecuación final, sus puntajes B, los pesos Beta, el error estándar de estimación que aportó cada una a la B, la contribución de cada una de las variables independientes al modelo a través de la prueba t de Student, así como su significancia. Únicamente se reportan las dimensiones del modelo que obtuvieron resultados estadísticamente significativos.

\section{Modelo psicosocial de RS en estudiantes del campus de la salud}

En la tabla tres se observa el modelo de regresión para la responsabilidad social en estudiantes del área de la salud. Se obtuvo una $\mathrm{Rm}=0.599$, una $\mathrm{R}^{2}=$ 0.359 y una $\mathrm{R}^{2}$ aj.=0.131; así, la responsabilidad social universitaria fue explicada en un $13.1 \%$ por las dimensiones independientes incluidas. El EE fue de 0.375 , de modo que, al predecir los puntajes de esta regresión, se tendrá un error de \pm 0.375 respecto al promedio. Se obtuvo una $\mathrm{F}_{(26.73)}=1.630 ; \mathrm{p}=$ 0.05. Las dimensiones con valor predictivo fueron ausencia de autoconcepto emotivo negativo autoafirmativo, autoconcepto social afiliativo y falta de apoyo social como estilo de enfrentamiento a los problemas. Lo anterior permite concluir que los universitarios del campus de salud que reportan mayor responsabilidad social son aquellos que no se consideran emotivos negativos autoafirmativos, que se consideran sociales afiliativos y que no suelen usar el apoyo social al enfrentar sus problemas.

\section{Modelo psicosocial de Rs en estudiantes de ciencias sociales}

En la tabla 4 se observa el modelo de regresión para la RS en estudiantes del campus de ciencias sociales. Se obtuvo una $\mathrm{Rm}=0.656$, una $\mathrm{R}^{2}=0.431$ y una $\mathrm{R}^{2}$ aj. = 0.228; así, la RSU fue explicada en un $22.8 \%$ por las dimensiones independientes incluidas. El EE fue de 0.477 , de modo que, al predecir los puntajes de esta regresión, se tendrá un error de \pm 0.477 respecto al promedio. Se obtuvo una $\mathrm{F}_{(26.73)}=2.126 ; \mathrm{p}=$ 0.006. Las dimensiones con valor predictivo fueron directo revalorativo, preocupación empática e instrumental constructivo. Lo anterior permite concluir que los estudiantes del campus de ciencias sociales que reportan mayor RS son aquellos que enfrentan sus problemas de manera directa revalorativa, que se preocupan de manera empática por los demás y que consideran poseer un autoconcepto instrumental constructivo.

\section{Modelo psicosocial de RS en estudiantes de ciencias exactas}

En la tabla 5 se observa el modelo de regresión para la responsabilidad social en estudiantes del campus de ciencias exactas. Se obtuvo una $\mathrm{Rm}=0.684$, una $\mathrm{R}^{2}$ $=0.467$ y una $\mathrm{R}^{2}$ aj. = 0.278; así, la responsabilidad social universitaria fue explicada en un $27.8 \%$ por las dimensiones independientes incluidas. El EE fue de 0.430 , de modo que, al predecir los puntajes de esta regresión, se tendrá un error de \pm 0.430 respecto al promedio. Se obtuvo una $\mathrm{F}_{(26.73)}=2.465 ; \mathrm{p}=0.001$. 
La única dimensión con valor predictivo fue instrumental constructivo. Lo anterior permite concluir que los universitarios del campus de ciencias exactas que reportan mayor responsabilidad social son aquellos con autoconcepto instrumental constructivo.

Tabla 3. Análisis de regresión múltiple para predecir la RSU en estudiantes del área de la salud

\begin{tabular}{|l|c|c|c|c|c|}
\hline \multicolumn{1}{|c|}{ Dimensión } & B & EE & Beta & t & p \\
\hline $\begin{array}{l}\text { Ausencia de autoconcepto } \\
\text { emocional negativo autoafirmativo }\end{array}$ & -0.109 & 0.055 & -0.274 & 0.05 \\
\hline Autoconcepto social afiliativo & 0.105 & 0.044 & 0.338 & 2.371 \\
\hline Falta de uso de apoyo social & -0.079 & 0.033 & -0.297 & -2.385 & 0.02 \\
\hline
\end{tabular}

Fuente: elaboración propia.

Tabla 4. Análisis de regresión múltiple para predecir la RSU en estudiantes de ciencias sociales

\begin{tabular}{|l|c|c|c|c|c|}
\hline \multicolumn{1}{|c|}{ Dimensión } & B & EE & Beta & t & P \\
\hline Directo revalorativo & 0.230 & 0.081 & 0.409 & 2.827 & 0.006 \\
\hline Preocupación empática & 0.224 & 0.116 & 0.262 & 1.927 & 0.05 \\
\hline Instrumental constructivo & 0.107 & 0.055 & 0.225 & 1.922 & 0.05 \\
\hline
\end{tabular}

Fuente: elaboración propia.

Tabla 5. Análisis de regresión múltiple para predecir la RSU en estudiantes de ciencias exactas

\begin{tabular}{|c|c|c|c|c|c|}
\hline Dimensión & B & EE & Beta & $\boldsymbol{t}$ & $\boldsymbol{p}$ \\
\hline Instrumental constructivo & 0.147 & 0.050 & 0.310 & 2.943 & 0.004 \\
\hline
\end{tabular}

Fuente: elaboración propia 


\section{Modelo psicosocial de RsU en estudiantes de arquitectura, hábitat, arte y diseño}

En la tabla 6 se observa el modelo de regresión para la RSU en estudiantes del campus de arquitectura, hábitat, arte y diseño. Se obtuvo una $\mathrm{Rm}=0.634$, una $\mathrm{R}^{2}=0.403$ y una $\mathrm{R}^{2}$ aj. $=0.190$; así, la RSU fue explicada en un 19\% por las dimensiones independientes incluidas. El EE fue de 0.440, de modo que, al predecir los puntajes de esta regresión, se tendrá un error de \pm 0.440 respecto al promedio. Se obtuvo una $\mathrm{F}_{(26.73)}=1.892 ; \mathrm{p}=0.01$. La única dimensión con valor predictivo fue la ausencia de emocional negativo. Lo anterior permite concluir que los estudiantes de arquitectura, hábitat, arte y diseño que reportan mayor responsabilidad social son aquellos que enfrentan sus problemas sin la expresión emocional negativa.

\section{Modelo psicosocial de RSU en estudiantes de ciencias biológicas y agropecuarias}

Como se observa en la tabla 7, el análisis de regresión múltiple en estudiantes del campus de ciencias biológicas y agropecuarias obtuvo una $\mathrm{F}_{(26.73)}=1.034 ; \mathrm{p}=0.439$. A partir de lo anterior, se concluye que ninguna de las dimensiones estudiadas obtuvo un valor predictivo para la responsabilidad social en los estudiantes de este campus. Los modelos psicosociales resultantes se presentan de manera gráfica en la figura 2.

Tabla 6. Análisis de regresión múltiple para predecir la RSU en estudiantes de arquitectura, hábitat, arte y diseño

\begin{tabular}{|c|c|c|c|c|c|}
\hline Dimensión & $\boldsymbol{B}$ & $\boldsymbol{E E}$ & Beta & $\boldsymbol{t}$ & $\boldsymbol{p}$ \\
\hline Ausencia de emocional negativo & -0.152 & 0.060 & -0.340 & -2.530 & 0.01 \\
\hline
\end{tabular}

Fuente: elaboración propia.

Tabla 7. Análisis de regresión múltiple para predecir la RSU universitaria en estudiantes de ciencias biológicas y agropecuarias

\begin{tabular}{|c|c|c|c|c|c|c|}
\hline \multirow{2}{*}{ Campus } & & $\begin{array}{c}\text { Suma de } \\
\text { cuadrados }\end{array}$ & $\begin{array}{c}\text { Grados de } \\
\text { libertad }\end{array}$ & $\begin{array}{c}\text { Media } \\
\text { cuadrática }\end{array}$ & $\boldsymbol{F}$ \\
\hline \multirow{2}{*}{ Ciencias biológicas y agropecuarias } & Regresión & 4.510 & 26 & 0.173 & \multirow{2}{*}{1.034} & 0.439 \\
\cline { 2 - 7 } & Residual & 12.250 & 73 & 0.168 & & \\
\hline
\end{tabular}

Fuente: elaboración propia. 
Figura 2. Modelos psicosociales de responsabilidad social universitaria (RSU) en estudiantes de diferentes campus
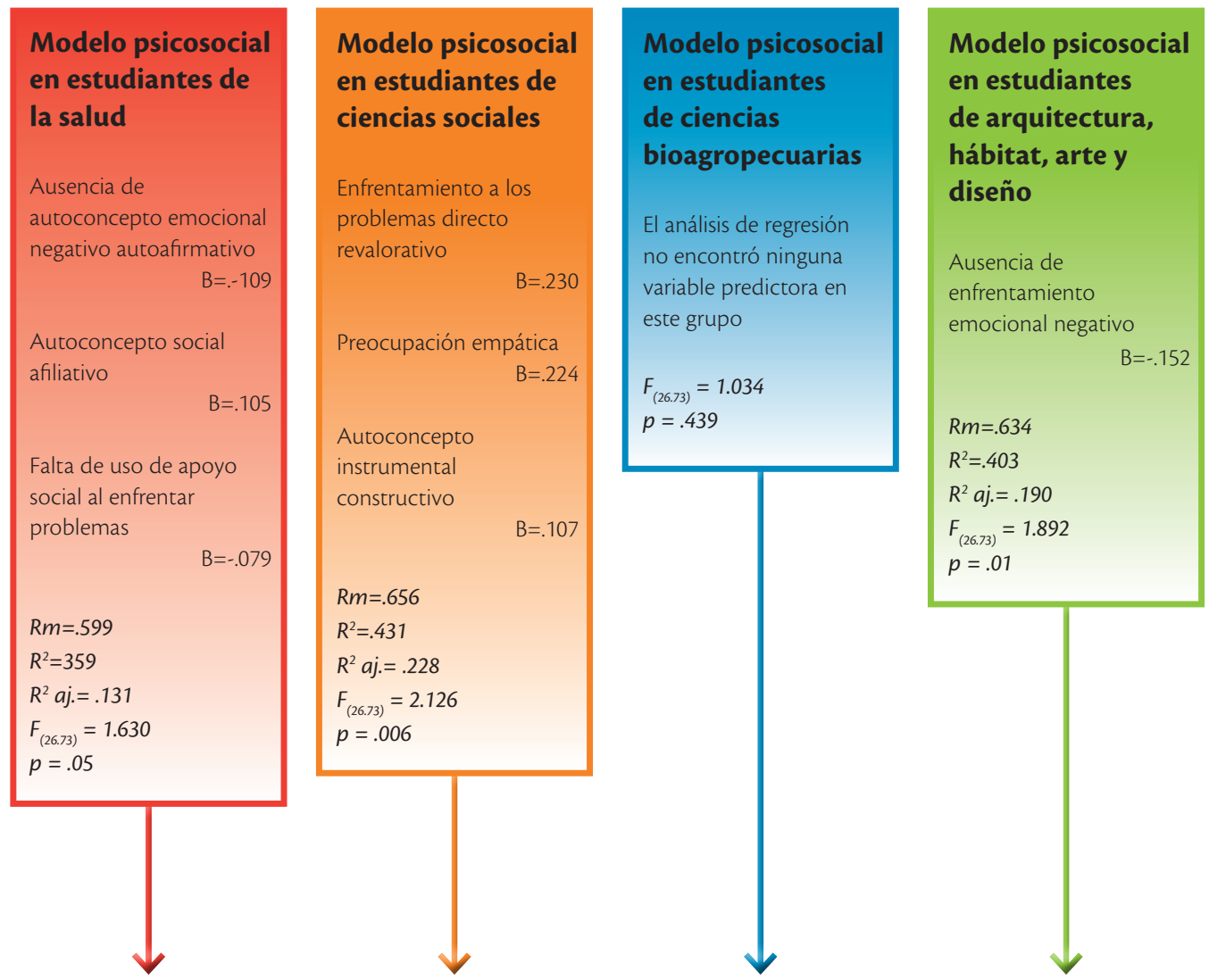
Modelo psicosocial en estudiantes de ciencias exactas
Autoconcepto
instrumental
constructivo
$R m=.684$
$R^{2}=.467$
$R^{2}$ aj. $=.278$
$F_{(26.73)}=2.465$
$p=.001$

\section{Responsabilidad Social Universitaria}




\section{Discusión}

Este trabajo tuvo como objetivo desarrollar modelos para explicar la RS en universitarios pertenecientes a diferentes campus a partir de una serie de variables psicosociales, tales como el locus de control, el autoconcepto, la empatía, el enfrentamiento a los problemas y la salud mental positiva. Adicionalmente, precisar si existen diferencias en cada una de las variables estudiadas entre los estudiantes de los diferentes campus.

Los resultados evidenciaron diferencias estadísticamente significativas en todas las variables contempladas en el estudio, aunque en todos los casos las diferencias fueron mínimas, pero estadísticamente significativas. Se observó que, en locus de control interno, la diferencia está a favor de los estudiantes de ciencias biológicas y agropecuarias, mientras que los estudiantes de ciencias exactas reportan mayor locus externo y mayor control de su vida en función de la simpatía y el manejo de los afectos. Es importante destacar las bajas puntuaciones obtenidas en el locus de control externo y en la afectividad como medio de control en la vida, así como el alto puntaje en el locus de control interno. Lo anterior permite observar la creencia de los universitarios en sus propias capacidades y en el esfuerzo personal como mediadores para el éxito, aspecto que se considera positivo en la juventud de hoy (Pinto, 2000; Serrano, 2003) y que es una característica mayormente observada en los estudiantes del campus de ciencias biológicas y agropecuarias de este estudio.

En autoconcepto, los estudiantes del campus de ciencias sociales se perciben como más expresivos y afiliativos en el plano social, así como ni mucho ni poco emocionales negativos; mientras que los que se perciben con mayor ética son los de ciencias biológicas y agropecuarias. Los datos anteriores permiten concluir que los estudiantes de este estudio cuentan con una imagen positiva de sí mismos y que tal imagen es acorde con la cultura mexicana generalmente descrita en términos de sociales y afiliativos
(Díaz-Guerrero, 2008; Díaz-Loving et al., 2002, Góngora y Reyes-Lagunes, 1999).

En cuanto a los estilos para enfrentar los problemas de la vida, solamente se encontró una diferencia por campus. La diferencia estuvo en el enfrentamiento emocional negativo, donde los estudiantes de ciencias sociales obtuvieron el puntaje más elevado. Sin embargo, este puntaje fue cercano a la media, de lo que se concluye un uso neutral de esta forma disfuncional de enfrentamiento a los problemas en los estudiantes de ciencias sociales, aspecto que se considera positivo; es decir, un bajo uso del enfrentamiento a los problemas en función de la expresión emocional negativa, se considera una dimensión saludable en las personas (Góngora et al., 2008).

Con respecto a la empatía, los estudiantes de ciencias sociales reportan mayor malestar emocional cuando están cerca de una persona en problemas. El malestar emocional constituye un indicador emocional de la empatía, aspecto que se considera adecuado como parte del perfil del universitario, sin embargo, esta dimensión tiene aspectos característicos en común con la angustia, en donde la persona con malestar emocional suele también reaccionar para mejorar su propio malestar, más que para ayudar a la persona en problemas (Martí, 2011). En teoría, esta dimensión de la empatía no estaría promoviendo comportamientos socialmente responsables. Por otro lado, los estudiantes del campus de ciencias biológicas y agropecuarias obtuvieron el puntaje más elevado en preocupación empática, por lo que se deduce que la capacidad para empatizar emocionalmente con los demás, es una característica que mejor describe a la empatía y que representa mejor a los estudiantes de este campus. Esta dimensión emocional de la empatía tiene el potencial de generar comportamientos socialmente responsables (Martí, 2011).

En cuanto a la salud mental positiva, el único factor que marcó una diferencia entre los estudiantes de los diferentes campus fue el autocontrol, en donde los estudiantes de ciencias exactas obtuvieron el puntaje 
más elevado. Al respecto, Lluch (1999: 246) considera que el autocontrol es una dimensión fundamental para el equilibrio emocional "un antídoto contra el estrés y un requisito básico para el funcionamiento adaptativo". Por tanto, el autocontrol constituye un factor promotor para la salud mental positiva, aspecto en el que particularmente destacan los estudiantes de ciencias exactas de este estudio. La salud mental positiva es una característica con el potencial de fortalecer individuos, grupos y comunidades, por lo que su promoción es importante (Castillo León, 2016).

Finalmente, respecto a la RSU, los estudiantes del campus de ciencias sociales se consideraron con las mayores capacidades de participación social, con mayores capacidades para gestionar socialmente su conocimiento y la mejor percepción de su universidad como una institución socialmente responsable. Asimismo, los estudiantes de ciencias biológicas y agropecuarias consideraron estar mejor formados de manera profesional y ciudadana. Si bien los estudiantes de ciencias sociales obtuvieron los mayores puntajes en tres de las cuatro dimensiones de la RSU, en realidad, los puntajes obtenidos por los estudiantes de los cinco campus fueron inferiores a la media, de lo cual se deduce que los estudiantes de este estudio no están tan seguros de sus capacidades para la gestión social de su conocimiento, para la participación social y de la imagen de su universidad como una institución que funciona de manera responsable. La única dimensión que obtuvo un puntaje superior a la media fue la formación profesional y ciudadana. Este puntaje elevado se observó en los estudiantes de los cinco campus siendo los del campus de ciencias biológicas y agropecuarias los que mostraron el puntaje más alto.

Lo anterior genera una contradicción en los resultados, por un lado, los estudiantes consideran que se están formando adecuadamente como profesionales y ciudadanos lo que, en teoría, debería también traducirse en la creencia en torno a sus habilidades para gestionar socialmente sus conocimientos, para la participación social y para considerar a su universidad como una institución responsable, pero no es el caso. Esta contradicción puede ser explicada tomando en cuenta los resultados de estudios previos en universitarios del sureste de México, donde se ha señalado que el concepto de responsabilidad social es todavía modesto en estudiantes yucatecos (Flores et al., 2016). Los resultados del presente estudio señalan que los universitarios únicamente se consideran socialmente responsables cuando analizan la contribución de su universidad para su formación profesional y ciudadana, sin embargo, todavía no consideran a la gestión social de su conocimiento (investigación, difusión y aplicación de su conocimiento), ni a la participación social (trabajo interdisciplinario, interinstitucional e intersectorial), ni la gestión responsable de su universidad como elementos que contribuyen a su responsabilidad social. Lo anterior genera una serie de interrogantes en la forma en la que se está enseñando y promoviendo la responsabilidad social en la universidad y en torno a la manera en la que se está conceptualizando entre los estudiantes.

Por otro lado, los modelos resultantes para explicar la responsabilidad social en los universitarios de los diferentes campus señalan lo siguiente. Para el campus de salud, la RSU se explicó a partir del autoconcepto y del enfrentamiento a los problemas. Es decir, los estudiantes del área de la salud socialmente responsables son los que no se consideran inconformes, impulsivos ni emocionalmente exaltados (ausencia de autoconcepto emotivo negativo autoafirmativo), por el contrario, se consideran poseedores de sentimientos positivos y de afecto por los demás (autoconcepto social afiliativo) y que al enfrentar los problemas de la vida no recurren al apoyo social (falta de enfrentamiento a través del apoyo social). Lo anterior señala que para explicar la responsabilidad social en los estudiantes del área de la salud basta con tener en cuenta la percepción que tienen de sí mismos (autoconcepto), así como la forma para enfrentar los problemas sin el apoyo social. Se puede observar que el 
modelo resultante para estudiantes de salud, excluye factores psicosociales que, a nivel conceptual, se consideran relacionados con la responsabilidad social (locus de control, autoconceptos instrumentales, autoconceptos éticos, empatía, enfrentamientos directos a los problemas y actitudes prosociales). Lo anterior genera una serie de interrogantes en torno a lo que significa y la forma como se practica la RS por parte de los estudiantes del área de la salud en donde, al parecer, basta con no ser inconforme, ni impulsivo, ni mostrar exaltación en las emociones; sentir afecto y cariño por el prójimo, así como la tendencia a resolver los problemas sin la ayuda de los demás, lo que "hace" a un universitario socialmente responsable. El modelo de RSU encontrado en universitarios del área de la salud contiene las variables psicosociales mayormente encontradas y, algunas veces, cuestionadas en profesionales del área de la salud (tendencia al trabajo individual y unidisciplinario, adecuación y conformidad a las normas del sistema institucional de salud, control excesivo de las emociones exaltadas ante los desafíos laborales, así como el afecto por los pacientes) (Castillo León, 2016; Rojas et al., 2010).

En el campus de ciencias sociales, la RSU se explicó a través del enfrentamiento a los problemas, la empatía y el autoconcepto. Es decir, los estudiantes de ciencias sociales con mayor responsabilidad social son los que enfrentan en forma directa los problemas y aprenden algo en el proceso (enfrentamiento directo revalorativo), generan respuestas afectivas, compasión y cariño ante las personas en dificultades (preocupación empática) y consideran poseer características que reflejan funcionalidad y habilidades en diferentes ámbitos (autoconcepto instrumental constructivo). El perfil obtenido para explicar la RSU en estudiantes de esta área está mucho más acorde con el marco conceptual del enfoque, en el cual se considera a la persona socialmente responsable como aquella que es competente en el plano profesional y ciudadano, lo que le permite generar, difundir y aplicar el conocimiento para promover el bien común así como para relacionarse con diferentes sectores de la sociedad para participar en la agenda de desarrollo (Arango et al., 2014; Martí et al., 2014; Vallaeys et al., 2009).

Por otro lado, tanto en los estudiantes de ciencias exactas como en los estudiantes de arquitectura, hábitat, arte y diseño, la RSU se explicó a partir de una sola de las variables psicosociales: el autoconcepto en el caso de los estudiantes de ciencias exactas, y el enfrentamiento a los problemas, en el caso de los estudiantes de arquitectura. Es decir, los estudiantes de ciencias exactas socialmente responsables son aquellos que poseen características que reflejan funcionalidad y habilidades en diferentes ámbitos (autoconcepto instrumental constructivo), mientras que en los estudiantes de arquitectura, hábitat, arte y diseño, la RSU se explicó mediante el enfrentamiento a los problemas, particularmente la ausencia de un enfrentamiento de tipo emocional negativo; así, los estudiantes de arquitectura enfrentan los problemas de la vida haciendo algo más que sólo quedarse con la expresión emocional que no lleva directamente a la solución del problema (ausencia de enfrentamiento emocional negativo).

Los modelos resultantes en ciencias exactas y en arquitectura son claros, la responsabilidad social universitaria se explica a partir de un yo visto en términos instrumentales (ciencias exactas) o a partir del enfrentamiento a los problemas con ausencia de emociones negativas (arquitectura, hábitat, arte y diseño). Sin embargo, en ambos modelos se observa una visión incompleta en torno a la responsabilidad social, la cual implica mucho más que sólo resolver los problemas desde la perspectiva individual y mucho más que sólo la regulación adecuada de las emociones. La responsabilidad social implica trabajo colaborativo muchas veces multi e interdisciplinario, resultado de una formación profesional que permite trascender las disciplinas de origen, que implica la vinculación y participación con diferentes sectores de la sociedad para el alcance de metas orientadas al bien común, así como implica la toma de conciencia 
de uno, del otro y del mundo particularmente en torno a las problemáticas que enfrenta. Es un proceso transformador que convierte a la persona en ciudadano del mundo (Arango et al., 2014; Martí et al., 2018; Rivera, 2018; Vallaeys et al., 2009). Los modelos resultantes en estudiantes de ciencias exactas y en estudiantes de arquitectura, hábitat, arte y diseño, en todo caso, parecen estar explicando cierto tipo de responsabilidad profesional más que de RSU.

Finalmente, el caso de los estudiantes de ciencias biológicas y agropecuarias es particular. Por un lado, se observa como el grupo con los puntajes más altos en locus de control interno, el grupo que se considera con mayor inteligencia socioemocional, con mayores rasgos éticos que los definen, con mayor preocupación empática y con el puntaje más alto en una de las dimensiones de RSU (la formación profesional y ciudadana). Sin embargo, los resultados del análisis de regresión múltiple revelaron que ninguna de las variables psicosociales estudiadas (autoconcepto, locus de control, enfrentamiento a los problemas, salud mental y empatía) pudo explicar la RSU en los estudiantes de este campus. Este resultado genera nuevas interrogantes en torno a lo que significa y la forma como se práctica la RSU por los estudiantes de este campus, la manera como es promovida por los docentes, así como la forma en la que puede ser explicada en esta área en particular. El desarrollo de futuras investigaciones desde la perspectiva cualitativa o la inclusión de nuevas variables de corte cuantitativo podrían aclarar el panorama. Además, se considera importante la generación de espacios adicionales de aprendizaje (asignaturas, talleres, seminarios) que faciliten a los estudiantes de todos los campus y particularmente a los de ciencias biológicas y agropecuarias, el desarrollo de las competencias para entrar en contacto con sus características psicosociales más positivas (empatía, internalidad, autoconcepto, enfrentamiento a los problemas y fortalezas en salud mental) y con ello potencializar sus comportamientos socialmente responsables.
De los diferentes modelos psicosociales elaborados para explicar la RSU, se puede observar lo siguiente. La fuerte contribución del autoconcepto para explicar la RS en los estudiantes de los diferentes $\mathrm{cam}^{-}$ pus, pues se observó la influencia de esta variable en estudiantes de ciencias de la salud, ciencias sociales y ciencias exactas. Un autoconcepto positivo juega un papel decisivo y central en el desarrollo de la personalidad, tal como lo destacan las principales teorías psicológicas; un autoconcepto positivo está en la base del adecuado funcionamiento personal, social y profesional del cual depende, en buena medida, la satisfacción personal (Esnaola et al., 2008). Asimismo, el fuerte lazo entre el autoconcepto y la responsabilidad social ha llevado a autores como Goñí y Fernández (2007) a proponer que éste es parte de un tipo particular de autoconcepto al cual denominan autoconcepto social, el cual se asocia de manera significativa con numerosos rasgos psicológicos, tales como la estabilidad emocional, la sociabilidad y la responsabilidad (Esnaola et al., 2008).

Una segunda variable que explica la responsabilidad social es el enfrentamiento a los problemas, particularmente en estudiantes de ciencias de la salud, de ciencias sociales y de arquitectura. Ante el panorama actual de transformación y cambio, el enfrentamiento a los problemas resulta ser una habilidad clave. Así, las formas para enfrentar los problemas de la vida constituyen indicadores para el ajuste, el bienestar y como mediadores del estrésadaptación (Góngora, 2000; Serrano, 2003, Góngora et al., 2008).

Finalmente, la empatía logra explicar la responsabilidad social en estudiantes de ciencias sociales. $\mathrm{Al}$ respecto, Vallaeys et al. (2009) consideran que la RSU implica orientar la formación del estudiante hacia el desarrollo de su propia conciencia como un elemento importante en la solución de los problemas sociales, mediante acciones concretas que tomen en cuenta también al otro y su realidad, proporcionando competencias interpersonales para comprender a 
los demás, sentirse afectado y comprometido con las necesidades de su entorno. Del mismo modo, Arango et al. (2014) consideran que los comportamientos socialmente responsables y las conductas prosociales se producen en gran medida por el desarrollo empático y emocional del individuo y que, por tanto, la empatía es una de las variables psicológicas altamente implicadas en los comportamientos socialmente responsables. Un estudiante socialmente responsable es un individuo capaz de comprometerse, escuchar y ponerse en el lugar del otro. Es un ciudadano empático que se preocupa no sólo por su bienestar sino por el bienestar de todos los que lo rodean (Martí y Martí, 2010; Martí et al., 2014).

\section{Conclusiones}

A partir de los resultados obtenidos se concluye que existen diferencias significativas en todas las variables estudiadas entre los estudiantes de los diferentes campus. Asimismo, se encontró que la RSU de los alumnos de ciencias sociales se explicó a partir de su estilo para enfrentar los problemas, así como a la empatía y el autoconcepto que posean; mientras que, en el caso de los del área de la salud, se explicó en función de su autoconcepto y de la forma como enfrentan los problemas de la vida. En el caso de los universitarios de ciencias exactas, la RSU se explicó únicamente a partir de su autoconcepto; mientras que para los de arquitectura, hábitat, arte y diseño, se explicó únicamente a partir de los estilos

\section{Referencias}

Asociación Nacional de Universidades e Institutos de Educación Superior (ANUIES) (2018), Visión y acción 2030. Propuesta de la ANUIES para renovar la educación superior en México. Diseño y concertación de políticas públicas para impulsar el cambio social, http://www.anuies.mx/media/ docs/avisos/pdf/VISION_Y_ACGION_2030.pdf [Consulta: mayo de 2021]. que poseen para el enfrentamiento a sus problemas. En el caso de los estudiantes de bioagropecuarias, ninguna de las variables estudiadas pudo explicar su RSU. Asimismo, de los cuatro modelos resultantes en este estudio, el único modelo que estuvo acorde con el marco conceptual de la RSU fue el encontrado en universitarios del campus de ciencias sociales.

Finalmente, la temática de la RSU constituye una de las principales prioridades para las IES pues el enfoque contribuye a la generación del capital humano y social que se traduce en mayor ciudadanía, democracia, participación social, desarrollo sostenible, justicia social y ciencia con conciencia. Por tanto, es importante mantener y aumentar la investigación en torno al tema. Por otro lado, los resultados del presente estudio confirman la aportación de las principales variables psicosociales (autoconceptos positivos, enfrentamientos funcionales a los problemas y la empatía) a la responsabilidad social del universitario. Asimismo, los modelos psicosociales resultantes generan nuevas interrogantes para futuras investigaciones en las cuales se sugiere la inclusión de diferentes variables (empoderamiento, motivación al logro, asertividad) con el objeto de analizar su contribución para la RSU. Del mismo modo, se sugiere la inclusión y adecuación de asignaturas que contemplen la adquisición y fortalecimiento de diversas características psicosociales pues, como se ha demostrado, tienen el potencial para contribuir en la dimensión socialmente responsable de los universitarios.
Arango, Olber, Sandra Clavijo, Isabel Puerta y José Sánchez (2014), "Formación académica, valores, empatía y comportamientos socialmente responsables en estudiantes universitarios", Revista de la Educación Superior, vol. 43, núm. 169, pp. 89-105.

Barrera, Mónica y Mirta Flores (2015), “Construcción de una escala de salud mental positiva para adultos en 
población mexicana", Revista Iberoamericana de Diagnóstico y Evaluación, vol. 1, núm. 39, pp. 22-33.

Castillo León, María (ed.) (2016), Promoción de la salud desde las ciencias sociales. Sus conceptos y aplicaciones, Mérida, Yucatán, UADY/ANTAL.

Díaz-Guerrero, Rogelio (2008), La psicología del mexicano 2. Bajo las garras de la cultura, México, Trillas.

Díaz-Loving, Rolando, Isabel Reyes-Lagunes y Sofía Aragón (2002), "Autoconcepto: desarrollo y validación de un inventario etnopsicológico", Revista Iberoamericana de Diagnóstico y Evaluación Psicológica, vol. 13, núm. 1, pp. 29-54.

Domínguez, Julio y Claudio Rama (eds.) (2012), La responsabilidad social universitaria en la educación a distancia, Perú, Universidad Católica Los Andes Chimbote.

Esnaola, Igor, Alfredo Goñi y José Madariaga (2008), “El autoconcepto: perspectivas de investigación", Revista de Psicodidáctica, vol. 13, núm. 1, pp. 179-194.

Flores, Mirta, María Cortés, Issac Ortega y María Morales (2016), "Responsabilidad social universitaria desde la visión de los estudiantes de psicología”, en María Rivera, Ruth Vallejo, Ana Méndez y Fabiola González (eds.), Fóvenes, psicología y responsabilidad social universitaria, México, CUMEX/Grañén-Porrua, pp. 95-110.

Góngora, Elías (2000), "El enfrentamiento a los problemas y el papel del control: una visión etnopsicológica en un ecosistema con tradición", Tesis de Doctorado, México, Universidad Nacional Autónoma de México.

Góngora, Elías e Isabel Reyes-Lagunes (1999), "La estructura de los estilos de enfrentamiento: rasgo y estado en un ecosistema tradicional mexicano", Revista Sonorense de Psicología, vol. 13, núm. 2, pp. 3-14.

Góngora, Elías, Mirta Flores, María Pinto y Mario Serrano (2008), "Estilos de enfrentamiento, asertividad y bienestar subjetivo en personas con síntomas depresivos", La psicología social en México, vol. 7, pp. 447-454.

Goñí, Eider (2009), "El autoconcepto personal: estructura interna, medida y variabilidad", Tesis de doctorado, España, Universidad del País Vasco.

Goñí, Eider y Arantza Fernández (2007), "Los dominios social y personal del autoconcepto", Revista Psicodidáctica, vol. 12, núm. 2, pp. 179-194.
Larrán-Jorge, Manuel y Francisco-Javier Andrades-Peña (2015), "Análisis de la responsabilidad social universitaria desde diferentes enfoques teóricos", Revista Iberoamericana de Educación Superior, vol. 6, núm. 15, pp. 91-107.

Lluch, María (1999), "Construcción de una escala para evaluar la salud mental positiva", Tesis de doctorado, Barcelona, Universidad de Barcelona.

Mayorá-Pernía, Carlos y Nelly Fernández (2015), "Locus de control y rendimiento académico en educación universitaria: una revisión bibliográfica", Revista Electrónica Educare, vol. 19, núm. 3, pp. 1-23, DOI: http://dx.doi.org/10.15359/ree.19-3.16 .

Martí, Juan (2011), "Responsabilidad social universitaria: estudio acerca de los comportamientos, los valores y la empatía en estudiantes de universidades iberoamericanas", Tesis de doctorado, Valencia, Universidad de Valencia.

Martí, Juan, Osacar Licandro y Ricardo Gaete-Quezada (2018), "La responsabilidad social de la educación superior como bien común. Conceptos y desafios", Revista de la Educación Superior, vol. 43, núm. 14, pp.33-55.

Martí, Juan y Manuel Martí (2010), "La responsabilidad social: ¿una universidad empática?”, Revista Nou-Dice, vol. 362, núm. 4, http://www.uv.es/noudise/362.pdf [Consulta: mayo de 2021].

Martí, Juan José, Manuel Martí-Vilar y Gonzalo Almerich (2014), "Responsabilidad social universitaria: influencia de valores y empatía en la autoatribución de comportamientos socialmente responsables", Revista Latinoamericana de Psicología, vol. 46, núm. 3, pp. 160-168.

Martínez-Usarralde, María, Daniela Gil-Salom y Doris Macías-Mendoza (2019), "Revisión sistemática de responsabilidad social universitaria y aprendizaje servicio. Análisis para su institucionalización", Revista Mexicana de Investigación Educativa, vol. 24, núm. 80, pp. 149-172.

Nieves, María (2010), "Calidad académica y responsabilidad social: el aprendizaje servicio como puente entre dos culturas universitarias", en Miguel Martínez (ed.), Aprendizaje servicio y responsabilidad social de las universidades, Barcelona, Educación universitaria Octaedro/ICE-UB, pp. 27- 56. 
Olarte-Mejía, Diana Victoria y Leonardo Alberto RíosOsorio (2015), "Enfoques y estrategias de responsabilidad social implementadas en instituciones de educación superior. Una revisión sistemática de la literatura científica de los últimos 10 años", Revista de la Educación Superior, vol. 55, núm. 3, pp. 19-40.

Organización de Naciones Unidas (ONU) (2015), Transformar nuestro mundo: la agenda 2030 para el desarrollo sostenible, Asamblea General de Naciones Unidas, https:/ / undocs.org/es/A/RES/70/1 [Consulta: mayo de 2021].

Organización de Naciones Unidas para la Educación Ciencia y Tecnología (UNESCO) (2013), Situación educativa de América Latina y el Caribe. Hacia una educación para todos, UNESCO/PRELAC/SEP, http://www. unesco.org/new/fileadmin/MULTIMEDIA/ FIELD/Santiago/images/SITIED-espanol.pdf [Consulta: mayo de 2021].

Pinto, María (2000), "Predicción de la intención conductual del suicidio mediante la teoría de la acción razonada", Tesis de Maestría, Mérida, Facultad de Psicología-Universidad Autónoma de Yucatán.

Reyes-Lagunes, Isabel y Elías Góngora (1998), Escala multidimensional y multisituacional de enfrentamiento a los problemas, México, Unidad de Investigaciones Psicosociales, UNAM (Proyecto CONACyT 1084 P-H).

Rivera, Claudia (2018), "Sobre la función social del conocimiento humano mediante la vinculación y transferencia del conocimiento en América Latina", Revista de la Educación Superior, vol. 48, núm. 189, pp. 121-132.
Rodríguez, Juan (2012), "La responsabilidad social es inherente a la naturaleza y visión de la universidad", en Julio Domínguez y Claudio Rama (eds.), La responsabilidad social universitaria en la educación a distancia, Perú, Universidad Católica Los Andes Chimbote, pp. 13-37.

Rojas, María, María Castillo, Rebelín Echeverría y Julia Candila (2010), "Modelo metodológico para desarrollar equipos interdisciplinarios en atención primaria de la salud", Revista Interamericana en Psicología, vol. 44, núm. 1, pp. 176-186.

Ruiz-Corbella, Marta y Ernesto López-Gómez (2019), "La misión de la universidad en el siglo XXI: comprender su origen para proyectar su futuro", Revista de la Educación Superior, vol. 48, núm. 189 pp. 1-19.

Serrano, Mario (2003), "Suicidio: factores protectores y de riesgo en adolescentes de Mérida", Tesis de Maestría, Mérida, Facultad de Psicología-Universidad Autónoma de Yucatán.

Vallaeys, François (2014), "La responsabilidad social universitaria: un nuevo modelo universitario contra la mercantilización", Revista Iberoamericana de Educación Superior, vol. 5, núm. 12, pp. 105-117.

Vallaeys, François, Cristina de la Cruz y Pedro Sasia (2009), Responsabilidad social universitaria. Manual de primeros pasos, México, McGraw Hill/BID.

Velasco, Pedro, Sofía Rivera, Rolando Díaz-Loving e Isabel Reyes-Lagunes (2015), "Construcción y validación de una escala de locus de control-bienestar subjetivo", Psicología Iberoamericana, vol. 23, núm. 2, pp. 45-54.

\section{Cómo citar este artículo:}

Serrano-Pereira, Mario-Gerardo, María-Teresita Castillo-León y Emelia Hernández-Payán (2021), “Modelos psicosociales de responsabilidad social en universitarios de diferentes campus", Revista Iberoamericana de Educación Superior (RIES), vol. XII, núm. 35, pp. 49-69, DoI: https://doi.org/10.22201/iisue.20072872e.2021.35.1082 [Consulta: fecha de última consulta]. 